\title{
Traversing planes of learning
}

\author{
Gerry Stahl
}

Published online: 19 October 2012

(C) International Society of the Learning Sciences, Inc. and Springer Science+Business Media New York 2012

\section{Planes of learning in CSCL}

Learning, cognition and knowledge building can be analyzed at multiple units of analysis. For instance, analyses of CSCL are often conducted on one of three levels: individual learning, small-group cognition or community knowledge building. One can identify and analyze important processes taking place at each of these levels of description. This tripartite distinction is grounded in the practices of CSCL. With its focus on collaborative learning, CSCL naturally emphasizes providing support for dyads and small groups working together. In practice, CSCL small-group activities are often orchestrated within a classroom context by providing some initial time for individual activities (such as background reading or homework drill), followed by the small-group work, and then culminating in whole-class sharing of group findings. Thus, the typical classroom practices tend to create three distinguishable levels of activity. Often, the teacher sees the group work as a warm-up or stimulation and preparation for the whole-class discussion, facilitated directly by the teacher. Conversely, the importance of testing individual performance and valuing individual learning positions the group work as a training ground for the individual participants, who are then assessed on their own, outside of the collaborative context. In both of these ways, group cognition tends to be treated as secondary to either individual or community goals. By contrast, the role of intersubjective learning is foundational in Vygotsky (1930/1978), the seminal theoretical source for CSCL. Regardless of which is taken as primary, the three planes are actualized in CSCL practice, and the matter of their relative roles and connections becomes subsequently problematic for CSCL theory (Dillenbourg et al. 1996; Rogoff 1995; Stahl 2006).

While these different units, levels, dimensions or planes are intrinsically intertwined, research efforts generally focus on only one of them and current analytic methodologies are designed for only one. Furthermore, there is little theoretical understanding of how the different planes are connected. To the extent that researchers discuss the connections among levels, they rely upon commonsensical notions of

G. Stahl $(\bowtie)$

Drexel University, Philadelphia, PA, USA

e-mail: Gerry@ijCSCL.org 
socialization and enculturation-popularizations of traditional social science. There are few explicit empirical analyses of the connections, and-as we discovered at a workshop on this issue at ICLS 2012-it is even hard to find data that would lend itself to conducting such analyses.

The individual unit of analysis is the traditional default. This assumed approach is supported by widespread training of researchers in the standard methods of psychology and education. In the era of cognitive science, analysis made heavy usage of mental models and representations in the minds of individuals (Gardner 1985). With the "turn to practice" (Lave and Wenger 1991; Schatzki et al. 2001), the focus shifted to processes within communities-of-practice. Group cognition lies in the less-well-charted middle ground. It involves the semantics, syntactics and pragmatics of natural language, gestures, inscriptions, etc. The meaning-making processes of small-group interaction involve inputs from individuals, based on their interpretation of the on-going context (Stahl 2006, esp. Ch. 16). They also take into account the larger social/historical/cultural/linguistic context, which they can reproduce and modify.

Computer technologies play a central role in mediating the multi-level, intertwined problem-solving, content-acquiring and knowledge-building processes that take place in CSCL settings. From a CSCL perspective, innovative technologies should be designed to support this mediation. This involves considering within the design process of collaboration environments how to prepare groups, individuals and communities to take advantage of the designed functionality and to promote learning on all planes-e.g., through the provision of resources for teacher professional development, scripted collaboration activities and student curriculum.

\section{The theory of interconnected planes}

How are the major planes of learning connected; how can we connect investigations at different units of analysis? In Fig. 1, we see highway ramps or bridges used as resources for connecting road levels or landmasses. While we are more interested in conceptual connections between levels of learning, it may be helpful to consider the more intuitive physical case initially. A highway ramp or bridge often creates a possibility that did not otherwise exist for going from one level to another at a given point. To traverse from a local road to a limited-access expressway, one must first find an available on-ramp. To cross a river from

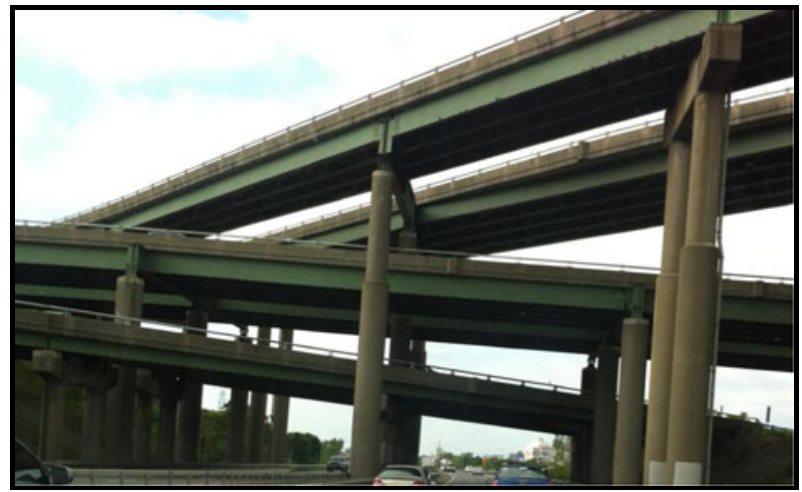

Fig. 1 Connecting ramps for the I-90 bridge across the Hudson River. Photo: G. Stahl, Albany, NY, 2012 
one side to the other, one may need a bridge. This is the individual driver's view. From a different vantage point - the perspective of the resource itself - the ramp or the bridge "affords" connecting the levels (Bonderup Dohn 2009).

By "affords," we do not simply mean that the connecting is a happy characteristic or accidental attribute of the bridge, but that the bridge, by its very nature and design, "opens up" a connection, which connects the banks of the river it spans. In his early work, Heidegger analyzed how the meaning of a tool was determined by the utility of the tool to the human user, within the network of meaning associated with that person's life and world; in his later writings, he shifted perspective to focus on things like bridges, paintings, sculptures, pitchers and temples in terms of how they themselves opened up new worlds, in which people could then dwell. In considering the intersubjective world in which collaboration takes place on multiple connected levels, we might say that the work of artifacts like bridges is to contribute the spanning of shores within the way that the world through which we travel together is opened up as a shared landscape of places and resources for meaningful discourse and action.

This transformation of perspective away from a human-centered or individual-mindcentered approach became characteristic for innovative theories in the second half of the 20th Century. It is a shift away from the individualistic, psychological view to a concern with how language, tools and other resources of our social life work. It is a post-cognitive move since it rejects the central role of mental models, representations and computations. The things themselves have effective affordances; it is not just a matter of how humans manipulate models in which the things are re-presented to the mind. In phenomenology, Husserl (1929/1960) called for a return to "the things themselves" (die Sache selbst) and Heidegger (1950/1967) analyzed "the thing" (das Ding)—such as the Alte Brücke of Heidelberg shown in Fig. 2-separate from our representation of it. In ethnomethodology, Garfinkel and Sacks (1970) followed Wittgenstein's (1953) linguistic turn to focus on the language games of words and the use of conversational resources (Stahl 2006, Ch. 18). In distributed cognition, Hutchins (1996) analyzed the encapsulation of historical cognition in technological instruments. In actor-network theory, Latour (1990) uncovered the agency of various kinds of objects in how they move across levels in enacting social transformations. Vygotsky (1930/1978) used the term "artifact" to refer to both tools and language as mediators of human cognition. The broader term "resource" is frequently used in sociocultural analysis (Furberg et al. Forthcoming; Linell 2001; Suchman 1987) for entities

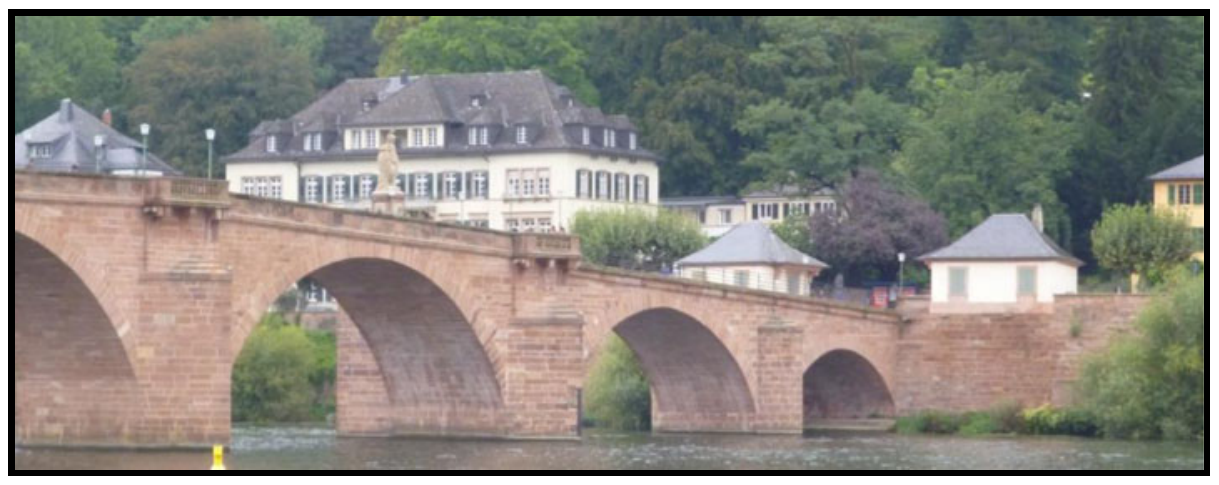

Fig. 2 The bridge across the Neckar River connecting the town with the residential hillside, as discussed by Heidegger (1950/1967). Photo: G. Stahl, Heidelberg, Germany, 2012 
referenced in discourse. Such artifacts or resources are identifiable units of the physical world (including speech and gesture) that are involved in meaning-making practicesbridging the classical mind/body divide.

A central research issue for CSCL is how collaborative knowledge building takes place. The main problem seems to be to understand the role of individual cognition and of societal institutions in small-group meaning-making processes. Figure 3 indicates (without claiming to explain or model) some typical processes on each of the primary planes of learning in CSCL and suggests possible paths of influence or connection, as events unfolding on the different planes interpenetrate each other. This figure is not meant to reify different levels or activities, but to sketch some of the constraints between different phenomena and possible flows of influence. The distinctions represented by boxes and arrows in the chart are intended to operationalize an infinitely complex and subtle matter for purposes of concrete analytic work by CSCL researchers.

Some researchers, such as many ethnomethodologists, argue against distinguishing levels. For instance, in their description of Conversation Analysis, Goodwin and Heritage (1990, p. 283) open their presentation with the following claim: "Social interaction is the primordial means through which the business of the social world is transacted, the identities of its participants are affirmed or denied, and its cultures are transmitted, renewed, and modified." Social interaction typically takes place in dyads and small groups, so interaction analysis is considered to be oriented to the small-group unit of analysis. However, CSCL researchers also want to analyze the levels of the individual and the culture as such-e.g., the individual identities and learning changes or the social practices and institutional forces:

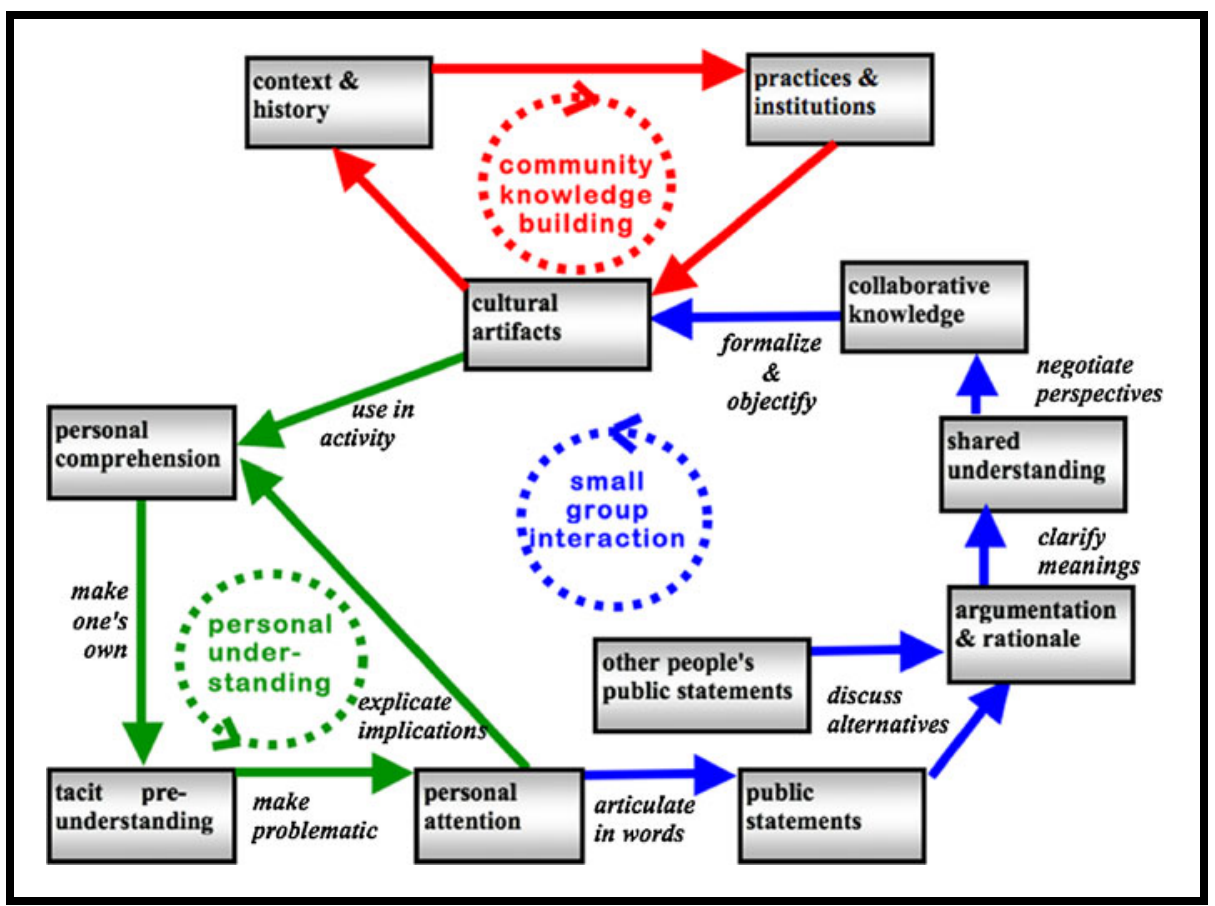

Fig. 3 A model of collaborative knowledge building. Adapted from (Stahl 2006, Ch. 9) 
How do the identities of participants get affirmed or denied as a result of social interaction? How are cultures transmitted, renewed and modified through social interaction?

In general, the sequential small-group interaction brings in resources from the individual, small-group and community planes and involves them in procedures of shared meaning making. This interaction requires co-attention to the resources and thereby shares them among the participants. Such a process may result in generating new or modified resources, which can then be retained at the various planes. The resources that are brought in and those that are modified or generated often take the form of designed physical artifacts and sedimented elements of language. We would like to study how this all happens concretely within data collected in CSCL settings.

\section{Analyses of connected planes}

This issue of $i j C S C L$ presents several studies that can be read in part within this problematic of traversing planes of learning. Each of the contributions offers some data from a CSCL setting, which can be analyzed in terms of the interpenetration of multiple planes of learning. In each case, a specific form of interactional resource is identified, which plays a mediating role in traversing the planes. Of course, these articles were drafted and submitted to the journal without any expectation that they would be read within this analytic problematic. So please appreciate each on its own terms, in relation to the framing content area and the stated findings. But it might also be interesting to consider them as case studies for understanding the relations among CSCL's three primary planes of learning.

In the first presentation, Jacques Lonchamp harkens back to the inaugural issue of ijCSCL, where Kienle and Wessner (2006) studied the evolution of the CSCL community through an analysis of conference papers. Lonchamp has previously appeared in this journal with theoretical contributions about mediating CSCL technologies, most recently applying the new theory of the instrumental genesis of technological artifacts to CSCL (Lonchamp 2006, 2009, 2012). Now, he tries to construct an account of this journal itself, as it has evolved over seven years. As we have argued, the journal has served as an important pillar of the CSCL research community (Stahl and Hesse 2006; Stahl et al. 2012). As such, the journal solicits the work of individual researchers - expressions of their personal investigations and thought. These ideas of individuals are inseparable from the group contexts within which they emerge and to which they are heavily targeted: the research labs, circles of corresponding specialists, traditions of shared paradigms. As analyses of journal and conference papers inevitably document, research thrives within an active and rapidly changing community or else it shrivels to irrelevance. As Latour and Woolgar (1979) showed some time ago, research is essentially mediated by concrete inscriptions; in ijCSCL, these take the form of published papers, with their references to other published papers. The journal paper is a key resource that traverses the planes of knowledge building in the CSCL community, and it can be analyzed and mapped as such.

The next paper shifts to another professional field, a medical specialty. Markus Nivala, Hans Rystedt, Roger Säljö, Pauliina Kronqvist and Erno Lehtinen provide a careful analysis of how certain resources in this world of work mediate collaborative reasoning. In particular, they are concerned with how innovative technical artifacts support the kind of co-attention in multi-modal settings that we have seen in other CSCL settings to be necessary to sustain collaboration (Çakir et al. 2009; Evans et al. 2011). The authors stress that their analysis must span the three planes of mental processes, the activity system and social interaction of the learning situation. They focus on "the interaction between individuals and how this 
interaction is mediated by language, referential practices and technology, which also constitute the unit of analysis." That is to say, they are concerned with how the small-group level of the interaction is mediated by the practices and artifacts that draw upon and simultaneously transform the personal skills and the medical institutions. Their analytic approach to referential practices as resources that span levels is ethnomethodologically informed (Stahl 2012).

The analysis of learning in a science museum by Susan A. Yoon, Karen Elinich, Joyce Wang, Christopher Steinmeier and Sean Tucker takes a quite different approach to studying the connection among levels. The learning domain is science, a community concerned with canonical theories. The learning intervention involves the use of augmented-reality tools and knowledge-building scaffolds within small groups of students visiting a museum. Here, the analytic focus of the experiment is on individual understanding: "CSCL environments are designed to be influenced by group interactions and we intentionally designed the conditions to understand how working in groups as a knowledge-building scaffold can impact understanding." This is typical for educational studies, which are concerned with learning outcomes of individual students. One can see in the analysis how community-level science content is mediated by the resources introduced at the small-group level to produce an effect at the individual level.

Similarly, the study by Chia-Ching Lin and Chin-Chung Tsai starts at a community level in order to investigate effects at the individual level. Rather than using group interaction to benefit individual outcomes, this intervention drew upon the collective intelligence of social media - specifically bookmarking - to benefit individual cognition. The experiment is in the area of information search. Traditional investigations of search focus on individual information behavior, while recent social informatics enquiries use Web 2.0 technologies to support search at the community level. Support for small-group search has unfortunately been under-researched (Stahl 2006, Ch. 7; Twidale and Nichols 1998). The bookmarks served as resources that bridged the collective and individual. The behaviors of the students in participating in the social activity were coded and correlated with the cognitive engagements of the students at the individual level.

The closing article of the 2012 volume of $i j C S C L$ considers CSCL scripting technology for orchestrating effective mixes of pedagogical activities at the individual, small-group and classroom planes. An important theme in the journal has been the definition of scripts for structuring interactions across levels. Here, Pericles Sobreira and Pierre Tchounikine explore an approach to supporting the efforts of teachers to adapt CSCL scripts to their particular classrooms. Scripts are resources for teachers, which operationalize catalogs of techniques for forming and using small groups and other classroom-organization approaches, such as a jig-saw script. Abstract technical discussions of scripting, including formalization and automation, can distance these potential resources from the practical world of the embedded teacher. The approach described here provides a clear representation of the design structure and envisioned effects of the script resource, so teachers can flexibly plan sequences of activities that traverse planes of learning

\section{References}

Bonderup Dohn, N. (2009). Affordances revisited: Articulating a Merleau-Pontian view. International Journal of Computer-Supported Collaborative Learning, 4(2), 151-170.

Çakir, M. P., Zemel, A., \& Stahl, G. (2009). The joint organization of interaction within a multimodal CSCL medium. International Journal of Computer-Supported Collaborative Learning, 4(2), 115-149. 
Dillenbourg, P., Baker, M., Blaye, A., \& O’Malley, C. (1996). The evolution of research on collaborative learning. In P. Reimann \& H. Spada (Eds.), Learning in humans and machines: Towards an interdisciplinary learning science (pp. 189-211). Oxford: Elsevier.

Evans, M. A., Feenstra, E., Ryon, E., \& McNeill, D. (2011). A multimodal approach to coding discourse: Collaboration, distributed cognition, and geometric reasoning. International Journal of ComputerSupported Collaborative Learning, 6(2), 253-278.

Furberg, A., Kluge, A., \& Ludvigsen, S. (Forthcoming). Students' conceptual sense-making with and of science diagrams in computer-based inquiry settings. International Journal of Computer-Supported Collaborative Learning, 8(1).

Gardner, H. (1985). The mind's new science: A history of the cognitive revolution. New York: Basic Books.

Garfinkel, H., \& Sacks, H. (1970). On formal structures of practical actions. In J. Mckinney \& E. Tiryakian (Eds.), Theoretical sociology: Perspectives and developments (pp. 337-366). New York: Appleton.

Goodwin, C., \& Heritage, J. (1990). Conversation analysis. Annual Review of Anthropology, 19, $283-307$.

Heidegger, M. (1950/1967). Das Ding. In Vorträge und Aufsätze II (pp. 37-60). Pfullingen: Neske.

Husserl, E. (1929/1960). Cartesian meditations: An introduction to phenomenology (D. Cairns, Trans.). The Hague: Martinus Nijhoff.

Hutchins, E. (1996). Cognition in the wild. Cambridge: MIT Press.

Kienle, A., \& Wessner, M. (2006). The CSCL community in its first decade: Development, continuity, connectivity. International Journal of Computer-Supported Collaborative Learning, 1(1), 9-33.

Latour, B. (1990). Drawing things together. In M. Lynch \& S. Woolgar (Eds.), Representation in scientific practice. Cambridge: MIT Press.

Latour, B., \& Woolgar, S. (1979). Laboratory life. Thousand Oaks: Sage Publications.

Lave, J., \& Wenger, E. (1991). Situated learning: Legitimate peripheral participation. Cambridge: Cambridge University Press.

Linell, P. (2001). Approaching dialogue: Talk, interaction and contexts in dialogical perspectives. New York: Benjamins.

Lonchamp, J. (2006). Supporting synchronous collaborative learning: A generic, multi-dimensional model. International Journal of Computer-Supported Collaborative Learning, 1(2), 247-276.

Lonchamp, J. (2009). A three-level analysis of collaborative learning in dual-interaction spaces. International Journal of Computer-Supported Collaborative Learning, 4(3), 289-317.

Lonchamp, J. (2012). An instrumental perspective on CSCL systems. International Journal of ComputerSupported Collaborative Learning, 7(2), 211-237.

Rogoff, B. (1995). Sociocultural activity on three planes. In B. Rogoff, J. Wertsch, P. del Rio, \& A. Alvarez (Eds.), Sociocultural studies of mind (pp. 139-164). Cambridge: Cambridge University Press.

Schatzki, T. R., Knorr Cetina, K., \& von Savigny, E. (Eds.). (2001). The practice turn in contemporary theory. New York: Routledge.

Stahl, G. (2006). Group cognition: Computer support for building collaborative knowledge. Cambridge: MIT Press.

Stahl, G. (2012). Ethnomethodologically informed. International Journal of Computer-Supported Collaborative Learning, $7(1), 1-10$.

Stahl, G., \& Hesse, F. (2006). IjCSCL - a journal for research in CSCL. International Journal of ComputerSupported Collaborative Learning, 1(1), 3-7.

Stahl, G., Law, N., \& Hesse, F. (2012). An international research community. International Journal of Computer-Supported Collaborative Learning, 7(3), 341-345.

Suchman, L. (1987). Plans and situated actions: The problem of human-machine communication. Cambridge: Cambridge University Press.

Twidale, M., \& Nichols, D. (1998). Designing interfaces to support collaboration in information retrieval. Interacting with Computers, 10(2), 177-193.

Vygotsky, L. (1930/1978). Mind in society. Cambridge, MA: Harvard University Press.

Wittgenstein, L. (1953). Philosophical investigations. New York: Macmillan. 